\title{
INVERSE SEMIGROUPS WHOSE FULL INVERSE SUBSEMIGROUPS FORM A CHAIN
}

\author{
by P. R. JONES $†$
}

(Received 6 January, 1980)

The structure of semigroups whose subsemigroups form a chain under inclusion was determined by Tamura [9]. If we consider the analogous problem for inverse semigroups it is immediate that (since idempotents are singleton inverse subsemigroups) any inverse semigroup whose inverse subsemigroups form a chain is a group. We will therefore, continuing the approach of $[5,6]$, consider inverse semigroups whose full inverse subsemigroups form a chain: we call these inverse $\nabla$-semigroups.

In $\$ 1$ we show that the non-trivial $\$$-classes of an inverse $\nabla$-semigroup form a chain, the associated principal factors being either cyclic or quasi-cyclic $p$-groups with zero ( $p$ a prime) or isomorphic to $B_{5}$, the five-element combinatorial Brandt semigroup. Inverse $\nabla$-semigroups are then characterized by these properties together with $(C)$ : for any non-idempotents $x$ and $y$ with $J_{x}<J_{y}, x=x x^{-1} y^{n}$ for some non-zero integer $n$.

In $\$ 2$ the property (C) is used to further elucidate the properties of inverse $\nabla$-semigroups. It is shown, for instance, that each element of such a semigroup $S$ has index at most 2 . If $S$ has an infinite subgroup $G$, then $G$ contains all the non-idempotents of $S$; if $S$ has a non-trivial subgroup $G$ of prime-power, but not prime, order, then $x^{2} \in G$ for every non-idempotent $x$ of $S$.

Meakin [8] described a very special class of inverse $\nabla$-semigroups: those with no proper inverse subsemigroups whatsoever. In the present paper the characterization given by the author in [6] of those inverse semigroups whose lattice of full inverse subsemigroups is distributive is clearly relevant.

1. A characterization. We begin with some terminology and notation, and a summary of the results from [5] which we will use. For background on lattices of full inverse subsemigroups the reader is referred to $[5,6]$.

Denote by $\mathscr{L} \mathscr{F}(S)$, or just $\mathscr{L} \mathscr{F}$, the lattice of full inverse subsemigroups of the inverse semigroup $S$ (that is, the lattice of those inverse subsemigroups of $S$ containing the semilattice $E$ of idempotents of $S$ ). If $A \subseteq S$, denote by $\langle A\rangle$ the inverse subsemigroup of $S$ generated by $A$, and by $\langle E, A\rangle$ the full inverse subsemigroup generated by $A$, that is, $\langle E \cup A\rangle$. In general we use the notation of [4].

Result 1.1 ([5, Corollary 1.2, Proposition 1.3]). For each $\Phi$-class $J$ of $S$ the relation $\gamma_{J}$ on $\mathscr{L F}$, defined by $A \gamma_{J} B$ if $A \cap J=B \cap J$, is a congruence. In the lattice of congruences on $\mathscr{L} \mathscr{F}, \wedge\left\{\gamma_{J}: J \in S / \mathscr{F}\right\}=0$. Hence $\mathscr{L} \mathscr{F}$ is a subdirect product of the lattices $\mathscr{L} \mathscr{F} / \gamma_{J}, J \in S / \mathscr{I}$. Moreover $\mathscr{L} \mathscr{F} / \gamma_{J} \cong \mathscr{L F}(\operatorname{PF}(J))$, where $\operatorname{PF}(J)$ is the principal factor associated with $J$, under the isomorphism $A \gamma_{J} \rightarrow(A \cap J) \cup\{0\}$.

$†$ The author gratefully acknowledges receipt of a Monash University Research Fellowship.

Glasgow Math. J. 22 (1981) 159-165. 
COROLlaRy 1.2. The lattice $\mathscr{L} \mathscr{F}$ is distributive if and only if $\mathscr{L F}(\operatorname{PF}(J))$ is distributive for each $\mathscr{I}$-class $J$.

Result 1.3 ([5, Corollary 3.6]). If $S$ is a completely 0-simple inverse semigroup with $\mathscr{L} \mathscr{F}$ distributive then $S$ is either a group with zero or is isomorphic to $B_{5}$, the combinatorial Brandt semigroup with five elements.

(Note: as in [5] we define $\operatorname{PF}(J)=J \cup\{0\}$, the product of two elements of $J$ being their product in $S$ if it lies in $J$, and all other products being zero. Thus $\operatorname{PF}(J)$ is always 0 -simple).

Finally, we require a characterization of $\nabla$-groups, (that is groups whose subgroups form a chain under inclusion).

Result 1.4 ([10, Theorem 5]). A group is a $\nabla$-group if and only if it is a cyclic or quasi-cyclic p-group, for some prime $p$.

(The notation $Z\left(p^{\infty}\right)$ is often used for quasi-cyclic $p$-groups. The reader is referred to [3] for their properties).

Since $\mathscr{L} \mathscr{F}\left(B_{5}\right)$ is a two-element chain (by Theorem 3.2 of [5]), it is immediate from Result 1.3 that the completely 0 -simple $\nabla$-semigroups are just $\boldsymbol{B}_{5}$ and the $\nabla$-groups with zero adjoined. We now show that these are the only 0 -simple inverse $\nabla$-semigroups; thus by Result 1.1 , every inverse $\nabla$-semigroup is completely semisimple.

Suppose $S$ is an inverse $\nabla$-semigroup which is 0 -simple but not completely 0 -simple. Clearly $\mathscr{L} \mathscr{F}(S)$ is distributive. It was shown in [6] that such a semigroup is in fact a simple semigroup $S^{*}(=S \backslash 0)$ with zero adjoined and that $\mathscr{L} \mathscr{F}\left(S^{*}\right) \cong \mathscr{L} \mathscr{F}(S)$. Further $S^{*}$ is $E$-unitary (that is $e x=e, e \in E$, implies that $x \in E$ ) and if $\sigma$ denotes the least group congruence on $S^{*}$ then the morphism $\sigma^{4}$ of $S^{*}$ upon $S^{*} / \sigma$ induces a lattice morphism of $\mathscr{L} \mathscr{F}\left(S^{*}\right)$ upon $\mathscr{L} \mathscr{F}\left(S^{*} / \sigma\right)$, the lattice of subgroups of $S^{*} / \sigma$. Since $S$ is a $\nabla$-semigroup, $S^{*} / \sigma$ is a $\nabla$-group, whence, by Result 1.4 , a $p$-group for some prime $p$. But this is impossible, for (since $S$ is not completely 0 -simple) $S^{*}$ contains an element of infinite order ([1, Theorem 2.54]), whose image in $S^{*} / \sigma$ again has infinite order.

We have proved the necessity of the property (B) in the following characterization of inverse $\nabla$-semigroups.

THEOREM 1.5. An inverse semigroup $S$ is a $\nabla$-semigroup if and only if

(A) the non-trivial $\Phi$-classes of $S$ form a chain,

(B) each non-trivial $\mathscr{f}$-class is either a cyclic or quasi-cyclic p-group for some prime $p$, or has principal factor isomorphic to $B_{5}$,

(C) for each $x, y \in S \backslash E_{S}$ with $J_{x}<J_{y}$ there is a non-zero integer $n$ such that $x=x x^{-1} y^{n}$.

Proof. Suppose $S$ is a $\nabla$-semigroup, and put $E=E_{\mathrm{s}}$. Let $x, y \in S \backslash E$. Either $\langle E, x\rangle \subseteq$ $\langle E, y\rangle$ so that $x \in\langle E, y\rangle$ and $J_{x} \leqslant J_{y}$, or $\langle E, y\rangle \subseteq\langle E, x\rangle$ so that $y \in\langle E, x\rangle$ and $J_{y} \leqslant J_{x}$. This proves (A); (B) has already been shown.

Again let $x, y \in S \backslash E$, with $J_{x}<J_{y}$. Clearly $y \notin\langle E, x\rangle$, so $x \in\langle E, y\rangle$. By expressing $x$ as a product involving $E$ and $y$ and permuting idempotents if necessary (c.f. Lemma 2.1 of 
[6]), we may write $x=e y^{n}$ for some $e \in E$ and non-zero integer $n$. Then $x x^{-1} \leqslant e$, so

$$
x=e y^{n}=x x^{-1} e y^{n}=x x^{-1} y^{n},
$$

proving (C).

Conversely, suppose $S$ is an inverse semigroup satisfying (A), (B) and (C), and let $A$, $B \in \mathscr{L} \mathscr{F}(S)$, where $A \neq E, B \neq E$. Suppose $A \nsubseteq B$ and let $a \in A \backslash B$. Put $J=J_{a}$. From (B) and the comments following Result 1.4, $\mathrm{PF}(J)$ is an inverse $\nabla$-semigroup. Now from Result 1.1 the map $C \rightarrow(C \cap J) \cup\{0\}$ is a lattice morphism of $\mathscr{L} \mathscr{F}(S)$ upon $\mathscr{L} \mathscr{F}(\operatorname{PF}(J))$. Hence since $a \in(A \cap J) \backslash(B \cap J)$, so that

$$
(A \cap J) \cup\{0\} \nsubseteq(B \cap J) \cup\{0\},
$$

we have

$$
(B \cap J) \cup\{0\} \subseteq(A \cap J) \cup\{0\},
$$

that is, $B \cap J \subseteq A \cap J$.

On the other hand if $b \in B \backslash E$ and $b \notin J$, then by (A), either $J_{b}>J$ or $J_{b}<J$. But if $J_{b}>J$ then, using (C), $a=\left(a a^{-1}\right) b^{n} \in B$, for some $n \neq 0$, a contradiction. Thus $J_{b}<J$ and, using (C) again, $b=\left(b b^{-1}\right) a^{n} \in A$, for some $n \neq 0$. Therefore $B \subseteq A$.

Hence $S$ is a $\nabla$-semigroup.

2. Some consequences. Throughout this section $S$ will be an inverse $\nabla$-semigroup, with $E=E_{s}$. The properties (A), (B) and (C) will be those in Theorem 1.5.

We consider first the restrictions that (B) places on a non-idempotent $x$ of $S$. Clearly if $x$ belongs to a subgroup, that is, $x$ has index 1 (in the terminology of $[4, \S 1.2]$ ), it has prime-power period. Suppose now that $x$ does not belong to a subgroup. Then $J_{x}$ has precisely 4 elements: $J_{x}=\left\{x, x^{-1}, x x^{-1}, x^{-1} x\right\}$. Consider the monogenic inverse subsemigroup $\langle x\rangle$ of $S$ : since $S$ is completely semisimple so is $\langle x\rangle$, and, further each non-group $\Phi$-class of $\langle x\rangle$ has at most four elements; from the description of all monogenic inverse semigroups (given in, for example, [2]) it is apparent that $x$ has index 2, that is, $x^{2}$ lies in the kernel $K_{x}$ of $\langle x\rangle$. From (B), again, $K_{x}$ is a (cyclic) group of order $p^{k}$, for some prime $p$ and some $\mathrm{k} \geqslant 0$. (If $k=0, K_{x}=\left\{x^{2}\right\}$ ). Thus

$$
x^{2}=x^{2+p^{k}}
$$

that is, $x$ has period $p^{k}$. The identity $f$ of $K_{x}$ is $x^{p^{k}}$ if $k \geqslant 1$, or $x^{2}$ if $k=0$.

Let $J$ be the $\mathscr{F}$-class of $S$ containing $K_{x}$. Let $z \in S$ be such that $z \mathscr{R} f$. Then, by (C), there is a non-zero integer $n$ such that $z=f x^{n}$. Thus $z \in K_{x}$. Thus $J$ is a group and in fact $J=K_{x}$. We have thus established

Propostrion 2.1. In an inverse $\nabla$-semigroup $S$ every element $x$ which is not in a subgroup of $S$ has index 2 and period $p^{k}$ for some prime $p$ and some $k \geqslant 0$. The kernel $K_{x}$ of $\langle x\rangle$ is an entire group g-class of $S$, with identity $x^{\mathrm{p}^{k}}$ if $k \geqslant 1$, or $x^{2}$ if $k=0$.

It is easily verified that any monogenic inverse semigroup generated by an element of index 2 and period $p^{k}, k \geqslant 0$, satisfies (A), (B) and (C) and is therefore a $\nabla$-semigroup. 
We now show that the property $(C)$ imposes major restrictions on the permissible combinations of non-trivial $\$$-classes of $S$. First, however, a technical lemma, whose proof is routine, is needed.

LEMMA 2.2. Let $T$ be any inverse semigroup, $G$ a group $\Phi$-class of $T$, with identity $e$, and $U$ an inverse subsemigroup of $T$ such that $e \leqslant f$ for all $f \in E(U)$. Then the map $u \mapsto e u$ is a morphism of $U$ into $G$.

Now let $G$ and $H$ be non-trivial group $g$-classes of $S$ with identities $e$ and $f$, respectively, such that $e<f$ (so $G<H$ as $g$-classes). From (C) it follows that the morphism $\phi_{f, e}: u \mapsto e u$ of $H$ into $G$, defined in the lemma, is surjective. In fact $K \phi_{f, e}=G$ for every non-trivial subgroup $K$ of $H$. In particular this implies $\operatorname{ker} \phi_{f, e}=\{f\}$, so $\phi_{f, e}$ is a bijection.

Furthermore $H$, being a cyclic or quasi-cyclic $p$-group, certainly contains a subgroup of order $p$. Thus $|G|=|H|=p$. Applying (A) we therefore have

Propostrion 2.3. If $S$ is an inverse $\nabla$-semigroup with more than one non-trivial maximal subgroup then there is a prime $p$ such that every non-trivial subgroup of $S$ has order precisely $p$.

Now let $J$ be a non-trivial $\Phi$-class of $S$ containing an element $x$ of index 2 , and let $G$ be a non-trivial group $g$-class of $S$, with identity $e$, such that $G<J$. The case $K_{\mathrm{x}}=G$ was covered in Proposition 2.1. By Exercise 3, \$8.4 of [1],e<xx,$e<x^{-1} x$ and so $e \leqslant\left(x x^{-1}\right)\left(x^{-1} x\right)=f$, the identity of $K_{x}$. Hence $K_{x} \geqslant G$ and there is a morphism of $\langle x\rangle$ upon $G$ (using (C)), as defined in Lemma 2.2, whose restriction to $K_{x}$ is the identity if $K_{x}=G$, and is the bijection $\phi_{f, e}$ defined above if $K_{x}>G$. (Note that the $\mathscr{f}$-class $K_{x}$ cannot be trivial, for if so, we have $x^{2}=x^{3}$, from which, using (C), it follows that $z^{2}=z^{3}$ for any $z \in G \backslash\{e\}$, a contradiction). Summing up, we have

Proposition 2.4. Let $x$ be an element of $S$ of index 2 and let $G$ be a group $\Phi$-class of $S$, with identity $e$, such that $G<J_{x}$. Then $G \leqslant K_{x}$ and the map $u \mapsto e u(u \in\langle x\rangle)$ is a morphism of $\langle x\rangle$ upon $G$ whose restriction to $K_{x}$ is a bijection upon $G$. Thus if $K_{x}$ is trivial so is every group $\Phi$-class $G<J_{x}$.

When the order of a group and a non-group $\$$-class is reversed the situation is rather different.

Proposirion 2.5. Let $x$ be an element of $S$ of index 2 and let $G$ be a non-trivial group $\Phi$-class of $S$, with identity $e$, such that $G>J_{x}$. Then $|G|=2$ and $x=x x^{-1} z$, where $z$ is the involution of $G$. In that case $x$ has period at most 2 .

Proof. Since $J_{x}<G$, we have $x=\left(x x^{-1}\right) z^{n}$ for some $n \neq 0$, for any $z \in G \backslash\{e\}$. Thus $x x^{-1}<e$ and for any such $z$,

$$
x x^{-1} z \mathscr{R} x x^{-1} e=x x^{-1} .
$$

But $R_{x x^{-1}}=\left\{x x^{-1}, x\right\}$ and if $x x^{-1} z=x x^{-1}$ then $x x^{-1} z^{n}=x x^{-1}$ for every non-zero integer $n$, contradicting (C). Hence $x x^{-1} z=x$ for every $z$ in $G \backslash\{e\}$. 
Suppose some element $z$ of $G$ has order $l>2$. Then

$$
x=x x^{-1} z=x x^{-1} z^{2},
$$

so $x z=x$ and $x z^{n}=x$ for all $n \geqslant 1$. But then

$$
x=x z^{l-1}=\left(x x^{-1} z\right) z^{l-1}=x x^{-1} z^{l}=x x^{-1} e=x x^{-1},
$$

a contradiction. So every non-identity element of $G$ has order 2 . Since $G$ is cyclic or quasi-cyclic, $|\mathrm{G}|=2$.

The last statement is an application of Propositions 2.3 and 2.1.

An interesting application of these results is the following.

COROLLARY 2.6. If an inverse $\nabla$-semigroup $S$ contains a quasi-cyclic maximal subgroup $G$, then $G$ constitutes the only non-trivial $\Phi$-class of $S$.

If $S$ contains a maximal subgroup $G$ of prime-power, but not prime order then $G$ is the only non-trivial maximal subgroup of $S$ and $G=K_{x}$ for every element $x$ of $S$ of index 2 .

Proof. First let $G$ be any non-trivial maximal subgroup of $S$ not of prime order. By Proposition 2.3, $G$ is the only non-trivial maximal subgroup of $S$. Suppose $x \in S$ has index 2. By Proposition 2.5, $G \ngtr J_{x}$. Thus $G<J_{x}$, so that by Proposition $2.4, G \leqslant K_{x}$ and $|G|=\left|K_{x}\right|$. Since $G$ is non-trivial, so is $K_{x}$. By Proposition $2.1, K_{x}$ is an entire $\Phi$-class of $S$ and hence $G=K_{x}$. Again by Proposition $2.1, K_{x}$ is finite so $G$ cannot be quasi-cyclic, proving the first statement.

We consider, finally, the relationship between two non-group $\mathscr{g}$-classes $J<J^{\prime}$ of $S$.

Let $x \in J \backslash E$ and $y \in J^{\prime} \backslash E$. From Proposition 2.1 we have that $K_{\mathrm{y}}$ is a group $\&$-class of $S$, so $K_{\mathrm{y}} \nsubseteq J$. Consider the case $K_{\mathrm{y}}>J$ and suppose $K_{\mathrm{y}}$ is trivial, that is, $K_{\mathrm{y}}=\left\{y^{2}\right\}$. From Exercise $3, \S 8.4$ of $[1]$ again, either $y^{2}>x x^{-1}$ or $y^{2}>x^{-1} x$. In the former case

$$
x x^{-1} y^{n}=x x^{-1}\left(y^{2} y^{n}\right)=x x^{-1}
$$

for any non-zero integer $n$, contradicting (C). Since the latter case similarly contradicts the obvious dual of (C), $K_{y}$ is therefore non-trivial. In fact, by Proposition $2.5,\left|K_{y}\right|=2$ and $x=x x^{-1} y^{3}$ (since $y^{3}$ is the involution in $K_{y}$ ), so that $x x^{-1}<y^{2}$ and

$$
x=\left(x x^{-1} y^{2}\right) y=x x^{-1} y .
$$

Suppose next that $K_{y}<J$. Then by Proposition $2.4, K_{y} \leqslant K_{x}$. From (C),

$$
x=x x^{-1} y^{ \pm 1}
$$

(since $J_{y^{n}}<J_{x}$ for $|n| \geqslant 2$ ). When $x=x x^{-1} y$, we obtain

$$
x^{2}=\left(x x^{-1} y x x^{-1} y^{-1}\right) y^{2},
$$

so $K_{y}=K_{x}$. We obtain a similar result when $x=x x^{-1} y^{-1}$. 


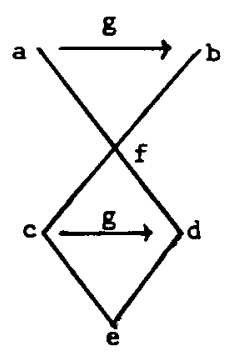

(a)

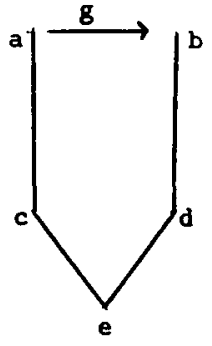

(b)

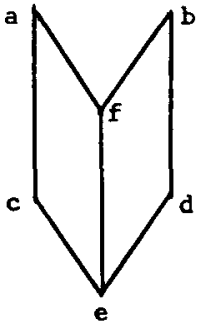

(c)

Figure 1

Finally suppose $K_{\mathrm{y}}$ and $J$ are incomparable. Then, by (A), we have that $K_{\mathrm{y}}$ is trivial, so that, by Proposition 2.4, $K_{x}$ is also. Again we have $x=x x^{-1} y^{ \pm 1}$. Summarizing,

Proposrtion 2.7. If $x$ and $y$ in $S$ have index 2 and $J_{x}<J_{y}$ then either

(i) $K_{y}>J_{x}$, in which case $\left|K_{y}\right|=2$ and $\left|K_{x}\right| \leqslant 2$, or

(ii) $K_{y}<J_{x}$, in which case $K_{y}=K_{x}$, or

(iii) $K_{y}$ and $J_{x}$ are incomparable, in which case $K_{x}$ and $K_{y}$ are trivial. In each case $x=x x^{-1} y^{ \pm 1}$.

Before continuing, we provide examples to show that each of these cases may occur.

First, let $E$ be the semilattice in Fig. 1(a), and let $G$ be a group of order 2, with $g$ its involution and 1 its identity. Let $G$ act on $E$ on the left by order automorphisms so that $g$ acts by "reflection". Let $U$ be the semidirect product of $E$ and $G$ : that is $U=E \times G$, with product

$$
(x, h)\left(x^{\prime}, h^{\prime}\right)=\left(x \wedge h x^{\prime}, h h^{\prime}\right) \text {. }
$$

(In the terminology of [7], $U=P(G, E, E)$ ). Then $U$ has 2 non-trivial group 8 -classes,

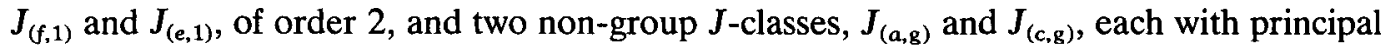
factor isomorphic with $\boldsymbol{B}_{5}$. Clearly (A) and (B) are satisfied and (C) is easily verified. Here $K_{(a, g)}>J_{(c, g)}$ and $\left|K_{(a, g)}\right|=\left|K_{(c, g)}\right|=2$. By taking the Rees quotient modulo $J_{(e, g)}$, we obtain a similar example with $\left|K_{(a, g)}\right|=2$ and $\left|K_{(c, g)}\right|=1$.

Now let $E$ be the semilattice in Fig. 1(b), let $G$ be as above, $g$ again acting by "reflection", and form the semidirect product $V$ of $E$ and $G$. In this case

$$
K_{(a, g)}=K_{(c, g)}=J_{(e, 1)} \text {. }
$$

(Here $\left|K_{(a, g)}\right|=2$ but examples may be similarly constructed where $K_{(a, g)}$ has arbitrary prime-power order).

Finally let $E$ be the semilattice in Fig. 1(c) and let $W$ be the full inverse subsemigroup of $T_{E}$ (see [4, Chapter V]) generated by the isomorphism $y$ taking $a E$ to $b E$ and fixing $f$. Then $W$ is an inverse $\nabla$-semigroup. If $x$ is the isomorphism taking $c E$ to $d E$ then it is easily verified that, if we take $S=W$, then $S$ has the properties described in Proposition 2.7.(iii).

We now continue the theme of Corollary 2.6. 
COROLlARY 2.8. If an inverse $\nabla$-semigroup $S$ contains a maximal subgroup of prime order $p \neq 2$, then every non-trivial subgroup has order $p$. Each element of $S$ of index 2 has the same kernel, $K$ say, and the same period p. Further $G \leqslant K$ for each non-trivial subgroup $G$ of $S$.

Proof. That every non-trivial subgroup has order $p$ follows from Proposition 2.3. Let $G$ be such a subgroup and let $x$ be any element of $S$ of index 2. By Proposition 2.5, $G \ngtr J_{x}$. Hence $G<J_{x}$, so by Proposition 2.4 we have that $G \leqslant K_{x}$ and $|G|=\left|K_{x}\right|$. So $x$ has period $p$.

If $y$ is another element of index 2 then $\left|K_{y}\right|=p$ also and we have $K_{y} \leqslant K_{x} \leqslant K_{y}$, that is, $K_{x}=K_{y}=K$, say, and $G \leqslant K$ for every non-trivial subgroup $G$ of $S$.

When $S$ has a maximal subgroup of order 2 then we can similarly show that every element of index 2 has period at most 2. However the examples above show that not every such element need have period 2 , and the kernels may be disjoint.

\section{REFERENCES}

1. A. H. Clifford and G. B. Preston, The algebraic theory of semigroups, Surveys of the Amer. Math. Soc. 7 (Providence, R.I., 1961 (Vol. I) and 1967 (vol. II)).

2. C. Eberhart, Elementary orthodox semigroups, Proc. Symposium on Inverse Semigroups and their Generalizations, Northern Illinois University, 1973, 23-33.

3. L. Fuchs, Abelian groups (Pergamon Press, 1960).

4. J. M. Howie, An introduction to semigroup theory, (Academic Press, 1976).

5. P. R. Jones, Semimodular inverse semigroups, J. London Math. Soc. 17 (1978), 446-456.

6. P. R. Jones, Distributive inverse semigroups, J. London Math. Soc. 17 (1978), 457-466.

7, D. B. McAlister, Groups, semilattices and inverse semigroups II, Trans. Amer. Math. Soc. 196 (1974), 351-370.

8. J. Meakin, One-sided congruences on inverse semigroups, Trans. Amer. Math. Soc. 206 (1975), 67-82.

9. T. Tamura, On a monoid whose submonoids form a chain, J. Gakugei Tokushima Univ. 5 (1954), 8-16.

10. T. Tamura, Commutative semigroups whose lattice of congruences is a chain. Bull. Soc. Math. France 97 (1969), 369-380.

Department of Mathematics

MONASH UNIVERSITY

Clayton, Victoria

Australia 3168
Present Address:

Department of Mathematics MARQUETTE UNIVERSTTY

MILWAUKEE

WISCONSIN 53233

USA 